\title{
COMPORTAMENTO RESILIENTE DE UM SOLO ARGILOSO DA REGIÃO DE VIÇOSA-MG NO ESTADO NATURAL E ESTABILIZADO COM CIMENTO E ALCATRÃO FRACIONADO ${ }^{1}$
}

\author{
Giovanni Levi Sant'Anna², Carlos Cardoso Machado $^{3}$, Carlos Alexandre Braz de Carvalho ${ }^{4}$, Dario Cardoso de \\ Lima $^{4}$ e Dalila Campos de Medeiros Fernandes ${ }^{6}$
}

\begin{abstract}
RESUMO - A importância do estudo das características resilientes dos solos e das misturas estabilizadas quimicamente justifica-se pela necessidade de conhecer o seu comportamento mecânico, sob a ação de cargas repetidas e transientes, quando constituintes do pavimento de estradas florestais. $\mathrm{O}$ método tradicional de determinação do módulo de resiliência, em laboratório, requer o emprego de um equipamento triaxial de cargas repetidas, que é relativamente complexo e caro, o que leva à necessidade de se desenvolver métodos mais simples para determinação do módulo de resiliência. Assim, buscou-se com este trabalho determinar o módulo de resiliência de um solo argiloso comum na região de Viçosa-MG em seu estado natural e quando estabilizado com cimento e alcatrão, como também propor correlações empíricas entre este e outros parâmetros geotécnicos de fácil obtenção em laboratório.
\end{abstract}

Palavras-chave: Estradas florestais, ensaio triaxial de cargas repetidas, módulo de resiliência e aditivos químicos.

\section{RESILIENT BEHAVIOR OF A CLAYEY SOIL FROM VIÇOSA-MG IN ITS NATURAL STATE AND AFTER STABILIZATION WITH CEMENT AND TAR}

\begin{abstract}
The study of the resilient response of soils and chemically stabilized mixtures is justified by the need to know the behavior of these soils under the action of repeated and transient loads. The traditional method for determining the resilient modulus of soils in laboratory requires complex and expensive repeated-loading triaxial equipment. Therefore, simpler methods should be developed. This work was carried out to identify the geotechnical and resilient properties of a clayey soil from Viçosa-MG in its natural state and after stabilization with cement and tar, and to propose empirical correlations between the resilient modulus of these materials and other geotechnical parameters, easily determined under laboratory conditions.
\end{abstract}

Key words: $\quad$ Forest roads, repeated-loading triaxial testing, resilient modulus and chemical additives.

\section{INTRODUÇÃO}

Apesar da existência de vários relatos na literatura internacional sobre o comportamento estático dos solos, das misturas solo-cal e das misturas solo-cimento, para fins rodoviários, observa-se que poucos estudos têm sido direcionados à análise da resposta dinâmica desses materiais e, em especial, das misturas solos-resíduos industriais (Sant'Anna et al., 2000).

Levando-se em consideração que o tipo de solo é de grande significância na avaliação do parâmetro reatividade solo-estabilizante, cresce o interesse em se desenvolver estudos de caráter regional para análise das propriedades dinâmicas de misturas estabilizadas

1 Recebido para publicação em 10.7.2002.

Aceito para publicação em 12.5.2003.

2 Mestre em Ciência Florestal, Departamento de Engenharia Florestal da Universidade Federal de Viçosa - UFV, 36571-000 Viçosa-MG, <ms25751@ correio.ufv.br >; ${ }^{3}$ Professor do Departamento de Engenharia Florestal da UFV; ${ }^{4}$ Professor do Departamento de Engenharia Civil da UFV, <cabraz@mail.ufv.br>; ${ }^{5}$ Professor do Departamento de Engenharia Civil da UFV, <DARIODELIMA@aol.com>; ${ }^{6}$ Doutora em Ciências Florestais pela UFV. 
quimicamente, particularmente em se considerando as aplicações no campo de estradas florestais. Os materiais que compõem o pavimento rodoviário estão submetidos a cargas dinâmicas e transientes, tornando indispensável que se proceda aos seus estudos em laboratório, também sob condições dinâmicas, tentando reproduzir, tanto quanto possível, as condições de campo (Sant'Anna et al., 2000).

No Brasil, a pouca experiência no que diz respeito à determinação das propriedades geotécnicas dinâmicas dos solos (Barros, 1997), principalmente quando se trata da obtenção em laboratório do módulo de resiliência de solos arenosos e argilosos, se deve, em grande parte, à escassez de equipamentos triaxiais dinâmicos nos centros técnicos de ensino e pesquisa em pavimentação, tanto aqui como no exterior.

Assim, Trindade et al. (2001), Carmo (1998), Lee et al. (1997) e Bernucci (1995), entre outros pesquisadores, propuseram, ao longo dos anos, correlações empíricas entre o módulo de resiliência e outros parâmetros geotécnicos de fácil obtenção.

Os objetivos deste trabalho foram determinar o módulo de resiliência de uma amostra de solo da microrregião de Viçosa-MG, em seu estado natural e estabilizada com alcatrão de madeira e cimento-alcatrão, para fins de pavimentação de estradas florestais, e propor correlações entre este e outros parâmetros geotécnicos de fácil obtenção em laboratório.

\section{2 . MATERIAL E MÉTODOS}

\subsection{Identificação dos Materiais}

Empregou-se um solo laterítico de grande ocorrência no relevo da cidade de Viçosa, localizada na Zona da Mata norte de Minas Gerais, Brasil. Este solo, aqui denominado ETA, é um Latossolo Vermelho-Amarelo, que se constitui no subleito de rodovias e terreno de fundação de construções civis; localiza-se nos topos de elevações com relevo plano; e apresenta um expressivo horizonte B profundo, poroso e bem drenado, com granulometria argilo-areno-siltosa, (60\% de argila, 22\% de areia e $18 \%$ de silte). Quanto à sua identificação, segundo o sistema de classificação de solos HRB, é um solo do grupo A-7-5(16). De acordo com a metodologia de classificação MCT, é um solo LG'. A amostra foi coletada no campus da Universidade Federal de Viçosa (UFV), no terço inferior de um talude de aproximadamente $6 \mathrm{~m}$ de altura, que se situa na rodovia MG 280, que liga as cidades de Viçosa e Paula Cândido, nas proximidades da Usina de Pré-Misturado a Frio da cidade de Viçosa.

O cimento empregado como estabilizante, no teor de $2 \%$ em relação ao peso de solo seco, foi do tipo Portland comercial CP-II, E-32, marca Barroso. O alcatrão utilizado, nos teores de 0,00, 0,25, 0,50 e 1,00\%, foi obtido da empresa V\&M Florestal, e na Universidade Federal de Viçosa passou por um tratamento térmico (fracionamento) a $170{ }^{\circ} \mathrm{C}$.

\subsection{Ensaios de Laboratório}

\subsubsection{Ensaios de Compactação}

Estes ensaios foram realizados com vistas à obtenção dos parâmetros peso específico seco máximo e teor ótimo de umidade do solo em seu estado natural e de suas misturas estabilizadas quimicamente, com base na NBR 7182/86 (Solo - Ensaio de Compactação), na energia do ensaio AASHTO Normal.

\subsubsection{Resistência à Compressão Simples}

Estes ensaios foram realizados em corpos-de-prova moldados da amostra do solo e nas misturas solo-cimentoalcatrão, em concordância com a metodologia preconizada por HEAD (1982), mas com aplicação da taxa de deformação de 1\% sugerida por LEE et al. (1997).

\subsubsection{Módulo de Resiliência}

Os módulos de resiliência do solo e das misturas solo-cimento-alcatrão foram determinados segundo a norma DNER-ME 131/94 (DNER, 1994), a partir dos resultados dos ensaios triaxiais de cargas repetidas, com instrumentação interna à câmara triaxial.

Para realização dos ensaios de compressão simples foram moldados corpos-de-prova do solo ETA, no estado natural e estabilizado com alcatrão e, ou, cimento, compactados na energia do ensaio AASHTO Normal. Foram moldados por processo dinâmico em três camadas iguais, considerando-se os seguintes limites fixados na preparação das amostras: $10 \pm 0,05 \mathrm{~cm}$; diâmetro: $5 \mathrm{~cm}$; teor ótimo de umidade $\pm 0,3 \%$; grau de compactação: $100 \pm 0,3 \%$. Os corpos-de-prova foram rompidos em uma prensa de compressão simples, com velocidade de subida 
do prato de $1 \mathrm{~mm} / \mathrm{min}$, como sugerido por LEE et al. (1997). Os corpos-de-prova do solo no estado natural foram levados a ruptura logo após a moldagem, e os corpos-deprova obtidos das misturas estabilizadas quimicamente foram submetidos à cura por 1, 7 e 28 dias. Para cada situação ensaiou-se um corpo-de-prova e determinou-se a curva tensão versus deformação do corpo-de-prova, obtendo-se os valores de tensão correspondente à deformação de $1 \%\left(\mathrm{~S}_{\mathbf{u} 1 \%}\right)$ e o módulo tangente inicial $\left(\mathrm{E}_{0}\right)$.

\subsection{Correlação para a Estimativa do Módulo de Resiliência dos Solos}

A publicação Aashto (1986) indica o emprego de ensaios mais simples para determinação do módulo de resiliência dos solos a partir do desenvolvimento de correlações entre esse parâmetro e ensaios-padrão de engenharia rodoviária.

Entretanto, é necessário observar que algumas das possíveis correlações podem não levar em consideração parâmetros importantes, como a dependência do módulo de resiliência com os níveis de tensão aplicados, o que acaba por restringir seu campo de emprego em estradas.

Como solução, tem-se procurado desenvolver correlações entre o módulo de resiliência e as propriedades índices dos solos, merecendo destaque a resiliência nãoconfinada.

Neste trabalho, foram obtidos os valores das tensões correspondentes à deformação de $1 \%\left(\mathrm{~S}_{\mathrm{u} 1 \%}\right)$ e do módulo tangente inicial $\left(\mathrm{E}_{0}\right)$ para o solo ETA em seu estado natural e estabilizado quimicamente com alcatrão e cimento e buscou-se estabelecer correlações estatísticas entre esses valores e os módulos de resiliência correspondentes e as variações nos níveis de tensão.

\section{RESULTADOS E DISCUSSÃO}

\subsection{Compactação}

No presente trabalho pode-se observar que a inclusão do alcatrão e do cimento ao solo causou variações no seu peso específico seco máximo $\left(\gamma_{\text {dmáx }}\right)$ e no teor ótimo de umidade $\left(\mathrm{W}_{\mathrm{ot}}\right)$. Verificou-se uma tendência de queda no teor ótimo de umidade e um ligeiro aumento no peso específico seco máximo das misturas solo-alcatrão e solocimento-alcatrão, o que pode ser constatado no Quadro 1.
Quadro 1 - Parâmetros de compactação das misturas solo-alcatrão e solo-cimento-alcatrão: teor ótimo de umidade $\left(W_{\text {ót }}\right)$ e peso específico seco máximo $\left(\gamma_{\mathrm{dmax}}\right)$

Table 1 - Compaction parameters of soil-tar and soilcement-tar mixtures: optimum water content $\left(W_{o ́ t}\right)$ and maximum dry density $\left(\gamma_{\text {dmáx }}\right)$

\begin{tabular}{|c|c|c|c|c|}
\hline \multirow{2}{*}{$\begin{array}{c}\text { Alcatrão } \\
(\%)\end{array}$} & \multicolumn{2}{|c|}{ Misturas Solo- Alcatrão } & \multicolumn{2}{|c|}{$\begin{array}{c}\text { Misturas Solo- Cimento- } \\
\text { Alcatrão }\end{array}$} \\
\cline { 2 - 5 } & $\mathrm{W}_{\text {ót }}$ & $\begin{array}{c}\gamma_{\mathrm{dmáx}} \\
\left(\mathrm{kN} / \mathrm{m}^{3}\right)\end{array}$ & $\begin{array}{c}\mathrm{W}_{\text {ót }} \\
(\%)\end{array}$ & $\begin{array}{c}\gamma_{\mathrm{dmáx}} \\
\left(\mathrm{kN} / \mathrm{m}^{3}\right)\end{array}$ \\
\hline 0,00 & 31,8 & 13,8 & 30,8 & 14,26 \\
0,25 & 31,5 & 14,11 & 29,3 & 14,26 \\
0,50 & 30,6 & 14,15 & 29,4 & 14,30 \\
1,00 & 30,5 & 14,15 & 30,2 & 14,40 \\
\hline
\end{tabular}

Fonte: Fernandes (2000).

\subsection{Resistência à Compressão Simples}

Nos Quadros 2 e 3 estão os resultados dos ensaios de compressão simples, tensão correspondente à deformação de $1 \%\left(\mathrm{~S}_{\mathrm{u} 1 \%}\right)$ e módulo tangente inicial $\left(\mathrm{E}_{0}\right)$ das misturas nos períodos de cura de 1, 7 e 28 dias. Nas Figuras 1 e 2 estão os resultados dos ensaios de compressão simples (RCS) e tensão correspondente à deformação de $1 \%\left(\mathrm{~S}_{\mathrm{u} 1 \%}\right)$ e dos ensaios de compressão simples (RCS) e módulo tangente inicial $\left(\mathrm{E}_{0}\right)$, para misturas solo-alcatrão e solo-cimento-alcatrão, respectivamente.

De modo geral, constatou-se que a estabilização do solo com alcatrão ou com alcatrão acrescido de cimento, considerando um tempo de cura de 28 dias, apresenta influência significativa nos valores de resistência à compressão simples (RCS), tensão correspondente à deformação de $1 \%\left(\mathrm{~S}_{\mathrm{u} 1 \%}\right)$ e módulo tangente inicial $\left(\mathrm{E}_{0}\right)$. Os resultados são influenciados pelo teor de alcatrão e período de cura, podendo-se associar valores máximos dos parâmetros no teor de alcatrão de $0,25 \%$, aos 28 dias de cura.

\subsection{Módulo de Resiliência}

A condição de carregamento, o estado de tensão, a natureza do solo e a natureza das misturas estabilizadas quimicamente estão entre um grande número de fatores que podem causar variações no valor do módulo de resiliência (Parreira et al., 1998). A grande maioria dos modelos adotados em mecânica dos pavimentos foi desenvolvida com base na natureza do solo, com predomínio da tensão desvio ou da tensão confinante,

R. Árvore, Viçosa-M G, v.27, n.3, p.269-278, 2003 
Quadro 2 - Resultados dos ensaios de compressão simples (RCS), tensão correspondente à deformação de $1 \%$ ( $\mathrm{S}_{\mathrm{u} 1 \%}$ ) e módulo tangente inicial $\left(\mathrm{E}_{0}\right)$ para mistura solo-alcatrão

Table 2 - Unconfined compression testing data determined at peak and at $1 \%$ strain $\left(S_{u 1 \%}\right)$, and initial tangent modulus $\left(E_{0}\right)$ : soil-tar mixtures

\begin{tabular}{|c|c|c|c|c|c|c|c|c|c|}
\hline \multirow{3}{*}{$\begin{array}{c}\text { Alcatrão } \\
(\%)\end{array}$} & \multicolumn{9}{|c|}{ Período de Cura (Dia) } \\
\hline & \multicolumn{3}{|c|}{1} & \multicolumn{3}{|c|}{7} & \multicolumn{3}{|c|}{28} \\
\hline & $\begin{array}{l}\text { RCS } \\
(\mathrm{kPa})\end{array}$ & $\begin{array}{l}\mathrm{S}_{\mathrm{u} 1 \%} \\
(\mathrm{kPa})\end{array}$ & $\begin{array}{c}\mathrm{E}_{0} \\
(\mathrm{kPa})\end{array}$ & $\begin{array}{l}\text { RCS } \\
(\mathrm{kPa})\end{array}$ & $\begin{array}{l}\mathrm{S}_{\mathrm{u} 1 \%} \\
(\mathrm{kPa})\end{array}$ & $\begin{array}{c}\mathrm{E}_{0} \\
(\mathrm{kPa})\end{array}$ & $\begin{array}{l}\text { RCS } \\
(\mathrm{kPa})\end{array}$ & $\begin{array}{l}\mathrm{S}_{\mathrm{u} 1 \%} \\
(\mathrm{kPa})\end{array}$ & $\begin{array}{c}\mathrm{E}_{0} \\
(\mathrm{kPa})\end{array}$ \\
\hline 0,00 & 241 & 150 & 13.000 & - & - & - & - & - & - \\
\hline 0,25 & 228 & 150 & 17.000 & 251 & 150 & 16.000 & 386 & 380 & 30.000 \\
\hline 0,50 & 244 & 150 & 10.000 & 290 & 175 & 17.000 & 308 & 390 & 26.000 \\
\hline 1,00 & 229 & 150 & 15.000 & 279 & 170 & 13.000 & 297 & 400 & 24.000 \\
\hline
\end{tabular}

Quadro 3 - Resultados dos ensaios de compressão simples (RCS), tensão correspondente à deformação de $1 \%$ (S $\mathrm{S}_{\mathrm{u}} \%$ ) e módulo tangente inicial $\left(\mathrm{E}_{0}\right)$ para mistura solo-cimento-alcatrão

Table 3 - Unconfined compression testing data determined at peak and at $1 \%$ strain $\left(S_{u 1 \%}\right)$, and initial tangent modulus $\left(E_{0}\right)$ : soil-cement-tar mixtures

\begin{tabular}{|c|c|c|c|c|c|c|c|c|c|}
\hline \multirow{3}{*}{$\begin{array}{c}\text { Cimento (2\%) } \\
\text { Alcatrão } \\
(\%)\end{array}$} & \multicolumn{9}{|c|}{ Período de Cura (Dia) } \\
\hline & \multicolumn{3}{|c|}{1} & \multicolumn{3}{|c|}{7} & \multicolumn{3}{|c|}{28} \\
\hline & $\begin{array}{l}\text { RCS } \\
(\mathrm{kPa})\end{array}$ & $\begin{array}{c}\mathrm{S}_{\mathrm{u} 1 \%} \\
(\mathrm{kPa})\end{array}$ & $\begin{array}{c}\mathrm{E}_{0} \\
(\mathrm{kPa})\end{array}$ & $\begin{array}{l}\text { RCS } \\
(\mathrm{kPa})\end{array}$ & $\begin{array}{l}\mathrm{S}_{\mathrm{u} 1 \%} \\
(\mathrm{kPa})\end{array}$ & $\begin{array}{c}\mathrm{E}_{0} \\
(\mathrm{kPa})\end{array}$ & $\begin{array}{l}\mathrm{RCS} \\
(\mathrm{kPa})\end{array}$ & $\begin{array}{l}\mathrm{S}_{\mathrm{u} 1 \%} \\
(\mathrm{kPa})\end{array}$ & $\begin{array}{c}\mathrm{E}_{0} \\
(\mathrm{kPa})\end{array}$ \\
\hline 0,00 & 598 & 320 & 42.000 & 695 & 220 & 41.000 & 826 & 500 & 58.000 \\
\hline 0,25 & 685 & 260 & 41.000 & 796 & 380 & 70.000 & 1096 & 380 & 80.000 \\
\hline 0,50 & 564 & 360 & 42.000 & 736 & 400 & 68.000 & 865 & 390 & 61.000 \\
\hline 1,00 & 512 & 360 & 36.000 & 483 & 440 & 52.000 & 693 & 400 & 65.000 \\
\hline
\end{tabular}

dependendo se o solo é argiloso ou arenoso, respectivamente.

Com base nos resultados dos ensaios triaxiais de cargas repetidas, procurou-se avaliar o desempenho do modelo sugerido no método do ensaio DNER-ME 131/ 94 para representação da variação do módulo de resiliência com o estado de tensão. Optou-se pelo modelo descrito pela equação 1 como a de melhor desempenho, tanto para o solo em seu estado natural quanto para o solo estabilizado quimicamente.

$$
\mathrm{M}_{\mathrm{R}}=\mathrm{k}_{2}+\mathrm{k}_{3}\left\{\left(\mathrm{k}_{1}-\left(\sigma_{1}-\sigma_{3}\right)\right\} ; \mathrm{k}_{1}>\left(\sigma_{1}-\sigma_{3}\right) ;\right.
$$

em que $M_{R}=$ módulo de resiliência $(\mathrm{MPa}) ; \sigma_{\mathrm{d}}=$ tensão desvio $(\mathrm{kPa})$; e $\mathrm{k}_{1}(\mathrm{kPa}) \mathrm{k}_{2}(\mathrm{kPa}) \mathrm{k}_{3}$ e $\mathrm{k}_{4}=$ parâmetros que variam de acordo com o material utilizado.

Os valores dos parâmetros $\mathrm{k}_{1}(\mathrm{kPa}) \mathrm{k}_{2}(\mathrm{kPa}) \mathrm{k}_{3} \mathrm{e} \mathrm{k}_{4}$, obtidos a partir dos resultados dos ensaios triaxiais de cargas repetidas, para cada um dos materiais estudados, solo natural e misturas estabilizadas quimicamente, em diferentes teores de alcatrão e tempo de cura, estão apresentados nos Quadros 4, 5, 6, 7, 8 e 9.

\subsection{Correlações Obtidas entre o Módulo de Resiliên- cia e Parâmetros Geotécnicos de Fácil Obtenção}

Lee et al. (1997) determinaram a correlação entre o módulo de resiliência de três solos finos de subleito do estado de Indiana, Estados Unidos, e a resistência à compressão simples $\mathrm{S}_{\mathrm{U} 1 \%}$. Os autores determinaram uma correlação significativa $\left(R^{2}=0,97\right)$ entre os parâmetros $\mathrm{M}_{\mathrm{R}}$ e $\mathrm{S}_{\mathrm{U} 1 \%}$, apresentados na equação 2:

$$
M_{\mathrm{R}}(\mathrm{psi})=695,4\left(\mathrm{~S}_{\mathrm{u} 1 \%}\right)-5,93\left(\mathrm{~S}_{\mathrm{u} 1 \%}\right)^{2}
$$

Os autores concluíram que a tensão determinada a $1 \%$ de deformação no ensaio de compressão nãoconfinada é um bom indicador do módulo de resiliência. 

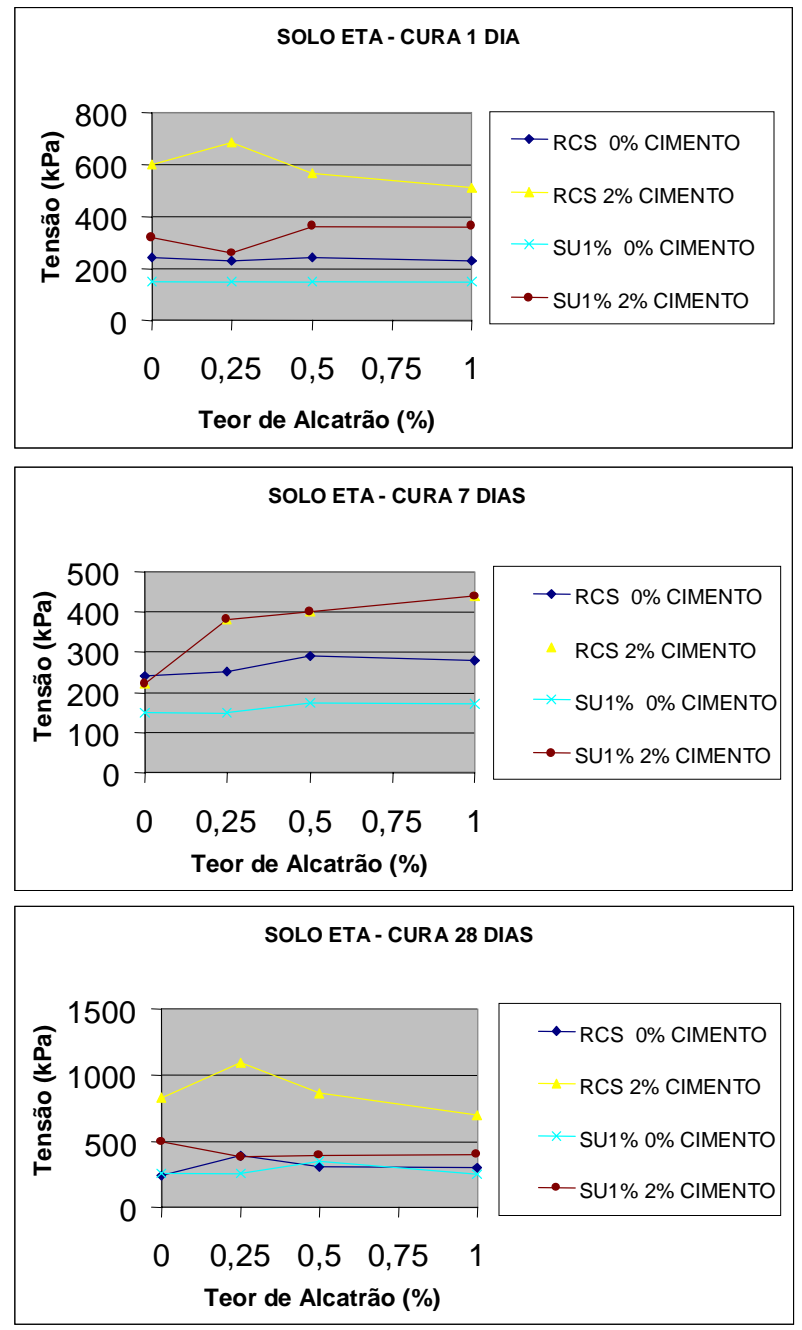

Figura 1 - Resultados dos ensaios de compressão simples (RCS) e tensão correspondente à deformação de $1 \%\left(\mathrm{~S}_{\mathrm{u1} \%}\right)$ para mistura solo-al catrão e solo-cimento-al catrão.

Figure 1 - Unconfined compression testing data determined at peak and at $1 \%$ strain $\left(\mathrm{S}_{\mathrm{u} 1 \%}\right)$ : soil-tar and soil-cementtar mixtures.

Carmo (1998) propôs duas equações para determinação do módulo de resiliência a partir do módulo tangente inicial determinado em ensaios de compressão não-confinada ( $\left.\mathrm{E}_{\mathrm{o}}, \mathrm{em} \mathrm{kPa}\right)$, da tensão desvio $\left(\sigma_{\mathrm{d},}\right.$ em $\left.\mathrm{kPa}\right)$ e do primeiro invariante de tensões $(\theta$, em $\mathrm{kPa})$. Para solos argilosos e arenosos têm-se, respectivamente, as relações apresentadas nas equações 3 e 4.

$\mathrm{M}_{\mathrm{R}}(\mathrm{kPa})=4.523,088\left(\mathrm{E}_{\mathrm{o}}\right)^{0,315821}\left(\sigma_{\mathrm{d}}\right)^{-0,343628}(\theta)^{0,419295}$
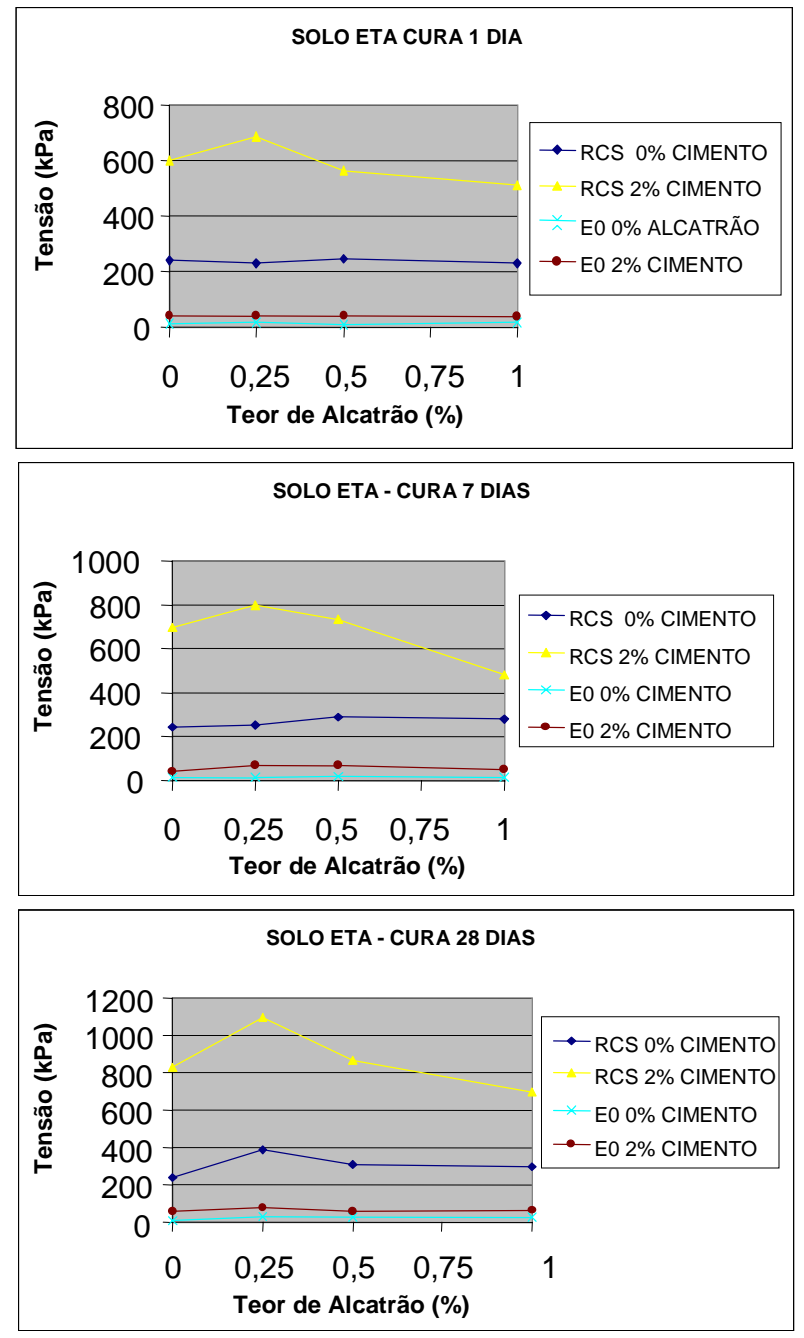

Figura 2 - Resultados dos ensaios de compressão simples (RCS) e módulo tangente inicial $\left(E_{0}\right)$ para mistura soloalcatrão e solo-cimento-alcatrão.

Figure 2 - Unconfined compression testing data and initial tangent modulus (EO): soil-tar and soil-cement-tar mixtures.

$\mathrm{M}_{\mathrm{R}}(\mathrm{kPa})=458,8053\left(\mathrm{E}_{\mathrm{o}}\right)^{0,491871}\left(\sigma_{\mathrm{d}}\right)^{0,542580}(\theta)^{-2,33031}$

Os parâmetros obtidos pelos resultados dos ensaios de compressão simples [tensão correspondente à deformação de $1 \%\left(\mathrm{~S}_{\mathrm{u} 1 \%}\right)$ e módulo tangente inicial $\left(\mathrm{E}_{0}\right)$ ] guardam estreita relação com o módulo de resiliência, uma vez que estas também são obtidas no ramo elástico da curva tensão versus deformação obtida no ensaio de compressão não-confinada, o que leva a crer serem promissoras as tentativas de correlações entre estes parâmetros e o módulo de resiliência.

R. Árvore, Viçosa-M G, v.27, n.3, p.269-278, 2003 
Quadro 4 - Parâmetros obtidos através do ensaio triaxial de cargas repetidas para o solo ETA e as misturas solo-alcatrão, no período de cura de um dia

Table 4 - Parameters obtained from repeated-loading triaxial testing data: ETA soil and soil-tar mixtures, one day curing time

\begin{tabular}{|c|c|c|c|c|}
\hline \multicolumn{3}{|c|}{ Modelo Bi-Linear Adotado } & \multicolumn{2}{|c|}{$\begin{array}{ll}\mathrm{M}_{\mathrm{R}}=\mathrm{k}_{2}+\mathrm{k}_{3} \cdot\left(\mathrm{k}_{1}-\sigma_{\mathrm{d}}\right) & \mathrm{k}_{1}>\sigma_{\mathrm{d}} \\
\mathrm{M}_{\mathrm{R}}=\mathrm{k}_{2}+\mathrm{k}_{4}\left(\sigma_{\mathrm{d}}-\mathrm{k}_{1}\right) & \mathrm{k}_{1}<\sigma_{\mathrm{d}}\end{array}$} \\
\hline Teor de Alcatrão (\%) & $\varepsilon_{\mathrm{r}}(\%)$ & $\sigma_{\mathrm{d}}(\mathrm{kPa})$ & $\mathrm{M}_{\mathrm{R}}(\mathrm{MPa})$ & Parâmetros $\mathrm{k}_{1}, \mathrm{k}_{2}, \mathrm{k}_{3} \mathrm{e} \mathrm{k}_{4}$ \\
\hline \multirow{4}{*}{0,00} & 0,056 & 72 & 128,571 & \multirow{4}{*}{$\begin{array}{l}\mathrm{k}_{1}=130 \mathrm{kPa} \\
\mathrm{k}_{2}=73000 \mathrm{kPa} \\
\mathrm{k}_{3}=958 \\
\mathrm{k}_{4}=-79\end{array}$} \\
\hline & 0,110 & 104 & 94,545 & \\
\hline & 0,180 & 131 & 72,778 & \\
\hline & 0,240 & 168 & 70,000 & \\
\hline \multirow{4}{*}{0,25} & 0,058 & 76 & 131,034 & \multirow{4}{*}{$\begin{array}{l}\mathrm{k}_{1}=132,5 \mathrm{kPa} \\
\mathrm{k}_{2}=70000 \mathrm{kPa} \\
\mathrm{k}_{3}=1080 \\
\mathrm{k}_{4}=-292\end{array}$} \\
\hline & 0,200 & 150 & 75,000 & \\
\hline & 0,380 & 192 & 50,526 & \\
\hline & 0,420 & 205 & 48,810 & \\
\hline \multirow{4}{*}{0,50} & 0,060 & 75 & 125,000 & \multirow{4}{*}{$\begin{array}{l}\mathrm{k}_{1}=190 \mathrm{kPa} \\
\mathrm{k}_{2}=50000 \mathrm{kPa} \\
\mathrm{k}_{3}=652 \\
\mathrm{k}_{4}=-490\end{array}$} \\
\hline & 0,120 & 115 & 95,833 & \\
\hline & 0,180 & 145 & 80,556 & \\
\hline & 0,340 & 220 & 64,706 & \\
\hline \multirow{4}{*}{1,00} & 0,060 & 77 & 128,333 & \multirow{4}{*}{$\begin{array}{l}\mathrm{K}_{1}=135 \mathrm{kPa} \\
\mathrm{k}_{2}=80000 \mathrm{kPa} \\
\mathrm{k}_{3}=833 \\
\mathrm{k}_{4}=-49\end{array}$} \\
\hline & 0,120 & 117 & 97,500 & \\
\hline & 0,210 & 165 & 78,571 & \\
\hline & 0,290 & 220 & 75,862 & \\
\hline
\end{tabular}

Quadro 5 - Parâmetros obtidos através do ensaio triaxial de cargas repetidas para misturas solo-alcatrão, no período de cura de sete dias

Table 5 - Parameters obtained from repeated-loading triaxial testing data: soil-tar mixtures, seven day curing time

\begin{tabular}{|c|c|c|c|c|}
\hline \multicolumn{3}{|c|}{ Modelo Bi-Linear Adotado } & \multicolumn{2}{|c|}{$\begin{array}{l}M_{R}=k_{2}+k_{3} \cdot\left(k_{1}-\sigma_{d}\right) \\
M_{R}=k_{2}+k_{4}\left(\sigma_{d}-k_{1}\right)\end{array}$} \\
\hline Teor de Alcatrão (\%) & $\varepsilon_{\mathrm{r}}(\%)$ & $\sigma_{\mathrm{d}}(\mathrm{kPa})$ & $\mathrm{M}_{\mathrm{R}}(\mathrm{MPa})$ & Parâmetros $\mathrm{k}_{1}, \mathrm{k}_{2}, \mathrm{k}_{3}$ e $\mathrm{k}_{4}$ \\
\hline \multirow{4}{*}{0,25} & 0,024 & 80 & 333,333 & \multirow{4}{*}{$\begin{array}{l}\mathrm{k}_{1}=130 \mathrm{kPa} \\
\mathrm{k}_{2}=130000 \mathrm{kPa} \\
\mathrm{k}_{3}=4067 \\
\mathrm{k}_{4}=-688\end{array}$} \\
\hline & 0,070 & 120 & 171,429 & \\
\hline & 0,150 & 160 & 106,667 & \\
\hline & 0,280 & 210 & 75,000 & \\
\hline \multirow{4}{*}{0,50} & 0,040 & 80 & 200,000 & \multirow{4}{*}{$\begin{array}{l}\mathrm{k}_{1}=120 \mathrm{kPa} \\
\mathrm{k}_{2}=98000 \mathrm{kPa} \\
\mathrm{k}_{3}=2550 \\
\mathrm{k}_{4}=-383\end{array}$} \\
\hline & 0,090 & 110 & 122,222 & \\
\hline & 0,150 & 140 & 87,500 & \\
\hline & 0,240 & 180 & 75,000 & \\
\hline \multirow{4}{*}{1,00} & 0,040 & 83 & 207,500 & \multirow{4}{*}{$\begin{array}{l}\mathrm{k}_{1}=120 \mathrm{kPa} \\
\mathrm{k}_{2}=90000 \mathrm{kPa} \\
\mathrm{k}_{3}=3176 \\
\mathrm{k}_{4}=-253\end{array}$} \\
\hline & 0,120 & 120 & 100,000 & \\
\hline & 0,200 & 160 & 80,000 & \\
\hline & 0,340 & 220 & 64,706 & \\
\hline
\end{tabular}

R. Árvore, Viçosa-MG, v.27, n.3, p.269-278, 2003 
Quadro 6 - Parâmetros obtidos através do ensaio triaxial de cargas repetidas para as misturas solo-alcatrão, no período de cura de 28 dias

Table 6 - Parameters obtained from repeated-loading triaxial testing data: soil-tar mixtures, 28 day curing time

\begin{tabular}{|c|c|c|c|c|}
\hline \multicolumn{3}{|c|}{ Modelo Bi-Linear Adotado } & \multicolumn{2}{|c|}{$\begin{array}{ll}\mathrm{M}_{\mathrm{R}}=\mathrm{k}_{2}+\mathrm{k}_{3} \cdot\left(\mathrm{k}_{1}-\sigma_{\mathrm{d}}\right) & \mathrm{k}_{1}>\sigma_{\mathrm{d}} \\
\mathrm{M}_{\mathrm{R}}=\mathrm{k}_{2}+\mathrm{k}_{4}\left(\sigma_{\mathrm{d}}-\mathrm{k}_{1}\right) & \mathrm{k}_{1}<\sigma_{\mathrm{d}}\end{array}$} \\
\hline Teor de Alcatrão (\%) & $\varepsilon_{\mathrm{r}}(\%)$ & $\sigma_{\mathrm{d}}(\mathrm{kPa})$ & $\mathrm{M}_{\mathrm{R}}(\mathrm{MPa})$ & Parâmetros $\mathrm{k}_{1}, \mathrm{k}_{2}, \mathrm{k}_{3}$ e $\mathrm{k}_{4}$ \\
\hline \multirow{4}{*}{0,25} & 0,020 & 65 & 325,000 & \multirow{4}{*}{$\begin{array}{l}\mathrm{k}_{1}=95 \mathrm{kPa} \\
\mathrm{k}_{2}=185000 \mathrm{kPa} \\
\mathrm{k}_{3}=4667 \\
\mathrm{k}_{4}=-614\end{array}$} \\
\hline & 0,042 & 90 & 214,286 & \\
\hline & 0,100 & 150 & 150,000 & \\
\hline & 0,150 & 190 & 126,667 & \\
\hline \multirow{4}{*}{0,50} & 0,039 & 80 & 205,128 & \multirow{4}{*}{$\begin{array}{l}\mathrm{k}_{1}=122,5 \mathrm{kPa} \\
\mathrm{k}_{2}=175250 \mathrm{kPa} \\
\mathrm{k}_{3}=703 \\
\mathrm{k}_{4}=-241\end{array}$} \\
\hline & 0,064 & 115 & 179,688 & \\
\hline & 0,082 & 140 & 170,732 & \\
\hline & 0,100 & 165 & 165,000 & \\
\hline \multirow{4}{*}{1,00} & 0,04 & 80 & 200,000 & \multirow{4}{*}{$\begin{array}{l}\mathrm{k}_{1}=97 \mathrm{kPa} \\
\mathrm{k}_{2}=160000 \mathrm{kPa} \\
\mathrm{k}_{3}=2353 \\
\mathrm{k}_{4}=-255\end{array}$} \\
\hline & 0,064 & 100 & 156,250 & \\
\hline & 0,090 & 135 & 150,000 & \\
\hline & 0,105 & 153 & 145,714 & \\
\hline
\end{tabular}

Quadro 7 - Parâmetros obtidos através do ensaio triaxial de cargas repetidas para as misturas solo-cimento e solocimento-alcatrão, no período de cura de um dia

Table 7 - Parameters obtained from repeated-loading triaxial testing data: soil-cement and soil-cement-tar mixtures, one day curing time

\begin{tabular}{|c|c|c|c|c|}
\hline \multicolumn{3}{|c|}{ Modelo Bi-Linear Adotado } & \multicolumn{2}{|c|}{$\begin{array}{ll}\mathrm{M}_{\mathrm{R}}=\mathrm{k}_{2}+\mathrm{k}_{3}\left(\mathrm{k}_{1}-\sigma_{\mathrm{d}}\right) & \mathrm{k}_{1}>\sigma_{\mathrm{d}} \\
\mathrm{M}_{\mathrm{R}}=\mathrm{k}_{2}+\mathrm{k}_{4}\left(\sigma_{\mathrm{d}}-\mathrm{k}_{1}\right) & \mathrm{k}_{1}<\sigma_{\mathrm{d}}\end{array}$} \\
\hline Teor de Alcatrão (\%) & $\varepsilon_{\mathrm{r}}(\%)$ & $\sigma_{\mathrm{d}}(\mathrm{kPa})$ & $\mathrm{M}_{\mathrm{R}}(\mathrm{MPa})$ & Parâmetros $\mathrm{k}_{1}, \mathrm{k}_{2}, \mathrm{k}_{3}$ e $\mathrm{k}_{4}$ \\
\hline \multirow{4}{*}{0,00} & 0,023 & 95 & 413,043 & \multirow{4}{*}{$\begin{array}{l}\mathrm{k}_{1}=170 \mathrm{kPa} \\
\mathrm{k}_{2}=300300 \mathrm{kPa} \\
\mathrm{k}_{3}=1503 \\
\mathrm{k}_{4}=-20\end{array}$} \\
\hline & 0,034 & 125 & 367,647 & \\
\hline & 0,060 & 180 & 300,000 & \\
\hline & 0,082 & 245 & 298,780 & \\
\hline \multirow{4}{*}{0,25} & 0,008 & 90 & $1.125,000$ & \multirow{4}{*}{$\begin{array}{l}\mathrm{k}_{1}=128 \mathrm{kPa} \\
\mathrm{k}_{2}=625000 \mathrm{kPa} \\
\mathrm{k}_{3}=13158 \\
\mathrm{k}_{4}=-290\end{array}$} \\
\hline & 0,017 & 120 & 705,882 & \\
\hline & 0,027 & 165 & 611,111 & \\
\hline & 0,039 & 232 & 594,872 & \\
\hline \multirow{4}{*}{0,50} & 0,010 & 100 & $1.000,000$ & \multirow{4}{*}{$\begin{array}{l}\mathrm{k}_{1}=124,5 \mathrm{kPa} \\
\mathrm{k}_{2}=750000 \mathrm{kPa} \\
\mathrm{k}_{3}=10204 \\
\mathrm{k}_{4}=-1454\end{array}$} \\
\hline & 0,015 & 120 & 800,000 & \\
\hline & 0,025 & 170 & 680,000 & \\
\hline & 0,036 & 220 & 611,111 & \\
\hline \multirow{4}{*}{1,00} & 0,020 & 89 & 445,000 & \multirow{4}{*}{$\begin{array}{l}\mathrm{k}_{1}=160 \mathrm{kPa} \\
\mathrm{k}_{2}=274900 \mathrm{kPa} \\
\mathrm{k}_{3}=2396 \\
\mathrm{k}_{4}=-157\end{array}$} \\
\hline & 0,035 & 125 & 357,143 & \\
\hline & 0,065 & 175 & 269,231 & \\
\hline & 0,095 & 240 & 252,631 & \\
\hline
\end{tabular}


Quadro 8 - Parâmetros obtidos através do ensaio triaxial de cargas repetidas para as misturas solo-cimento e solocimento-alcatrão, no período de cura de sete dias

Table 8 - Parameters obtained from repeated-loading triaxial testing data: soil-cement and soil-cement-tar mixtures, seven days curing time

\begin{tabular}{|c|c|c|c|c|}
\hline \multicolumn{3}{|c|}{ Modelo Bi-Linear Adotado } & \multicolumn{2}{|c|}{$\begin{array}{ll}\mathrm{M}_{\mathrm{R}}=\mathrm{k}_{2}+\mathrm{k}_{3} \cdot\left(\mathrm{k}_{1}-\sigma_{\mathrm{d}}\right) & \mathrm{k}_{1}>\sigma_{\mathrm{d}} \\
\mathrm{M}_{\mathrm{R}}=\mathrm{k}_{2}+\mathrm{k}_{4}\left(\sigma_{\mathrm{d}}-\mathrm{k}_{1}\right) & \mathrm{k}_{1}<\sigma_{\mathrm{d}}\end{array}$} \\
\hline Teor de Alcatrão (\%) & $\varepsilon_{\mathrm{r}}(\%)$ & $\sigma_{\mathrm{d}}(\mathrm{kPa})$ & $\mathrm{M}_{\mathrm{R}}(\mathrm{MPa})$ & Parâmetros $\mathrm{k}_{1}, \mathrm{k}_{2}, \mathrm{k}_{3}$ e $\mathrm{k}_{4}$ \\
\hline \multirow{4}{*}{0,00} & 0,012 & 80 & 666,667 & \multirow{4}{*}{$\begin{aligned} \mathrm{k}_{1} & =120 \mathrm{kPa} \\
\mathrm{k}_{2} & =255000 \mathrm{kPa} \\
\mathrm{k}_{3} & =10292 \\
\mathrm{k}_{4} & =-1178\end{aligned}$} \\
\hline & 0,022 & 100 & 454,545 & \\
\hline & 0,058 & 135 & 232,759 & \\
\hline & 0,092 & 175 & 190,217 & \\
\hline \multirow{4}{*}{0,25} & 0,012 & 100 & 833,333 & \multirow{4}{*}{$\begin{array}{l}\mathrm{k}_{1}=145 \mathrm{kPa} \\
\mathrm{k}_{2}=575000 \mathrm{kPa} \\
\mathrm{k}_{3}=5741 \\
\mathrm{k}_{4}=-2385\end{array}$} \\
\hline & 0,020 & 131 & 655,000 & \\
\hline & 0,028 & 155 & 553,571 & \\
\hline & 0,050 & 210 & 420,000 & \\
\hline \multirow{4}{*}{0,50} & 0,010 & 100 & $1.000,000$ & \multirow{4}{*}{$\begin{array}{l}\mathrm{k}_{1}=125 \mathrm{kPa} \\
\mathrm{k}_{2}=750000 \mathrm{kPa} \\
\mathrm{k}_{3}=10000 \\
\mathrm{k}_{4}=-1462\end{array}$} \\
\hline & 0,015 & 130 & 866,666 & \\
\hline & 0,025 & 175 & 700,000 & \\
\hline & 0,032 & 220 & 687,500 & \\
\hline \multirow{4}{*}{1,00} & 0,018 & 90 & 500,000 & \multirow{4}{*}{$\begin{array}{l}\mathrm{k}_{1}=125 \mathrm{kPa} \\
\mathrm{k}_{2}=345000 \mathrm{kPa} \\
\mathrm{k}_{3}=4429 \\
\mathrm{k}_{4}=-852\end{array}$} \\
\hline & 0,033 & 120 & 363,636 & \\
\hline & 0,040 & 134 & 335,000 & \\
\hline & 0,090 & 230 & 255,556 & \\
\hline
\end{tabular}

Quadro 9 - Parâmetros obtidos através do ensaio triaxial de cargas repetidas para as misturas solo-cimento e solocimento-alcatrão, no período de cura de 28 dias

Table 9 - Parameters obtained from repeated-loading triaxial testing data: soil-cement and soil-cement-tar mixtures, 28 day curing time

\begin{tabular}{|c|c|c|c|c|}
\hline \multicolumn{3}{|c|}{ Modelo Bi-Linear Adotado } & \multicolumn{2}{|c|}{$\begin{array}{ll}\mathrm{M}_{\mathrm{R}}=\mathrm{k}_{2}+\mathrm{k}_{3} \cdot\left(\mathrm{k}_{1}-\sigma_{\mathrm{d}}\right) & \mathrm{k}_{1}>\sigma_{\mathrm{d}} \\
\mathrm{M}_{\mathrm{R}}=\mathrm{k}_{2}+\mathrm{k}_{4}\left(\sigma_{\mathrm{d}}-\mathrm{k}_{1}\right) & \mathrm{k}_{1}<\sigma_{\mathrm{d}}\end{array}$} \\
\hline Teor de Alcatrão (\%) & $\varepsilon_{\mathrm{r}}(\%)$ & $\sigma_{\mathrm{d}}(\mathrm{kPa})$ & $\mathrm{M}_{\mathrm{R}}(\mathrm{MPa})$ & Parâmetros $\mathrm{k}_{1}, \mathrm{k}_{2}, \mathrm{k}_{3}$ e $\mathrm{k}_{4}$ \\
\hline \multirow{4}{*}{0,00} & 0,008 & 95 & $1.187,500$ & \multirow{4}{*}{$\begin{aligned} \mathrm{k}_{1} & =140 \mathrm{kPa} \\
\mathrm{k}_{2} & =720000 \mathrm{kPa} \\
\mathrm{k}_{3} & =10389 \\
\mathrm{k}_{4} & =-343\end{aligned}$} \\
\hline & 0,013 & 120 & 923,077 & \\
\hline & 0,022 & 155 & 704,545 & \\
\hline & 0,035 & 240 & 685,714 & \\
\hline \multirow{4}{*}{0,25} & 0,007 & 90 & $1.285,714$ & \multirow{4}{*}{$\begin{array}{l}\mathrm{k}_{1}=160 \mathrm{kPa} \\
\mathrm{k}_{2}=1025000 \mathrm{kPa} \\
\mathrm{k}_{3}=3724 \\
\mathrm{k}_{4}=-719\end{array}$} \\
\hline & 0,012 & 133 & $1.108,333$ & \\
\hline & 0,016 & 163 & $1.018,750$ & \\
\hline & 0,023 & 225 & 978,261 & \\
\hline \multirow{4}{*}{0,50} & 0,010 & 100 & $1.000,000$ & \multirow{4}{*}{$\begin{array}{l}\mathrm{k}_{1}=124 \mathrm{kPa} \\
\mathrm{k}_{2}=745000 \mathrm{kPa} \\
\mathrm{k}_{3}=10625 \\
\mathrm{k}_{4}=-1395\end{array}$} \\
\hline & 0,015 & 135 & 900,000 & \\
\hline & 0,025 & 180 & 720,000 & \\
\hline & 0,030 & 220 & 666,666 & \\
\hline \multirow{4}{*}{1,00} & 0,017 & 90 & 529,411 & \multirow{4}{*}{$\begin{array}{l}\mathrm{k}_{1}=140 \mathrm{kPa} \\
\mathrm{k}_{2}=285000 \mathrm{kPa} \\
\mathrm{k}_{3}=4300 \\
\mathrm{k}_{4}=-327\end{array}$} \\
\hline & 0,033 & 130 & 393,939 & \\
\hline & 0,050 & 165 & 330,000 & \\
\hline & 0,077 & 230 & 298,701 & \\
\hline
\end{tabular}

R. Árvore, Viçosa-MG, v.27, n.3, p.269-278, 2003 
Obtidos os valores das tensões correspondentes à deformação de $1 \%\left(\mathrm{~S}_{\mathbf{u} 1 \%}\right)$ e do módulo tangente inicial $\left(\mathrm{E}_{0}\right)$ para o solo ETA em seu estado natural e estabilizado quimicamente com alcatrão e cimento, buscou-se estabelecer correlações estatísticas entre esses valores e os módulos de resiliência correspondentes e as variações nos níveis de tensão. Um coeficiente de determinação $\left(R^{2}\right)$ igual a 0,825 representou o resultado mais significativo obtido ao considerar o solo ETA em seu estado natural e estabilizado com cimento e alcatrão como um único conjunto de dados, quando se correlacionou o módulo de resiliência com o primeiro invariante de tensões, com o módulo tangente inicial e com a tensão desvio, conforme apresentado na equação 5 e na Figura 3.

$$
\mathrm{M}_{\mathrm{R}}=\frac{5636 \mathrm{E}_{0}^{1,328646} \sigma_{d}^{1,697031}}{\theta^{3,07205}}
$$

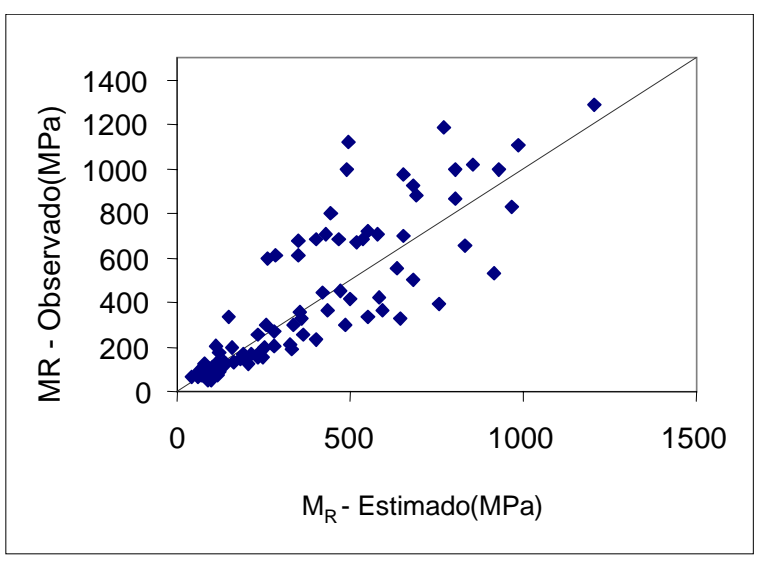

Figura $3-M_{R}$ - observado versus $M_{R}$ - estimado, considerando dados do solo ETA em seu estado natural e estabilizado quimicamente.

Figure 3 - Observed - $M_{R}$ versus estimated - $M_{R}$, based on ETA soil in its natural state and chemically stabilized mixtures.

Os parâmetros analisados mostraram-se indicadores significativos do módulo de resiliência, o que reforça a importância de proceder ao desenvolvimento de outras correlações com vistas a facilitar a determinação do módulo de resiliência dos materiais que compõem o pavimento e o subleito de estradas florestais.

\section{CONCLUSÕES}

Com base nos valores dos coeficientes de determinação $\left(\mathrm{R}^{2}\right)$, encontrados para algumas correlações do módulo de resiliência com os parâmetros de tensão correspondente à deformação de $1 \%$ e módulo tangente inicial, dos solos e, ou, misturas estabilizadas quimicamente, observou-se a ocorrência de variação significativa na resistência à compressão não-confinada e nos parâmetros elásticos do solo ETA após a adição de alcatrão e alcatrão e cimento. $\mathrm{O}$ teor de alcatrão e o período de cura mostraram-se fatores de importância nessas variações medidas.

Portanto, concluiu-se também que:

- a partir da análise dos dados apresentados nos Quadros 2 e 3 e nas Figuras 1 e 2, constatou-se que a adição de alcatrão às misturas solo-cimento mostrou-se benéfica para alguns tratamentos e encontram-se na faixa dos dados citados nas referências bibliográficas;

- há uma grande possibilidade de se determinar o módulo de resiliência através do emprego de alguns parâmetros geotécnicos de fácil obtenção, merecendo destaque a utilização da tensão correspondente à deformação de $1 \%$ e do módulo tangente inicial. Ambos os parâmetros ocorrem no ramo elástico das curvas tensão versus deformação e é justamente no regime elástico que o módulo de resiliência atua, avaliando a rigidez do material; e

- a correlação de mais de um parâmetro geotécnico com o módulo de resiliência também é bastante promissora, como ficou evidenciado neste trabalho, levando a crer na possibilidade de correlação de outros parâmetros geotécnicos com o módulo de resiliência.

\section{REFERÊNCIAS BIBLIOGRÁFICAS}

BARROS, J. M. C. Módulo de cisalhamento máximo de solos tropicais brasileiros. In: CONGRESSO NACIONAL DE GEOTECNIA, 6., 1997, Lisboa. Anais... Lisboa: v. 1, p. 117-126.

BERNUCCI, L. L. B. Considerações sobre o dimensionamento de pavimentos utilizando solos lateríticos para rodovias de baixo volume de tráfego. 1995. $237 \mathrm{f}$. Tese (Doutorado em Engenharia Civil) - Escola Politécnica, Universidade de São Paulo, São Paulo, 1995.

CARMO, C. A. T. A avaliação do módulo de resiliência através de ensaios triaxiais dinâmicos de dois solos compactados e a sua estimativa a partir de ensaios rotineiros. 1998. $131 \mathrm{f}$. Dissertação (Mestrado em Engenharia Civil) - Escola de Engenharia de São Carlos, São Carlos, 1998.

R. Árvore, Viçosa-MG, v.27, n.3, p.269-278, 2003 
DEPARTAMENTO NACIONAL DE ESTRADAS DE RODAGEM - DNER. Solos - Determinação do Módulo de Resiliência. São Paulo: 1994. 8 p. (Norma Rodoviária DNER-ME 131/94)

FERNANDES, D. C. M. Viabilidade do uso de alcatrão de madeira de eucalipto na estabilização de solos residuais para fins rodoviários. 2000. $124 \mathrm{f}$. Tese (Doutorado em Engenharia Florestal) - Universidade Federal de Viçosa, Viçosa, 2000.

HEAD, K. H. Manual of soil laboratory testing Permeability, shear strength and compressibility tests. New York: John Wiley \& Sons, 1982. v. 2. 412 p.
LEE, W. et al. Resilient Modulus of Cohesive Soils. ASCE. Journal of Geotechnical and Geoenvironmental Engineering, v. 123, n. 2, p. 131-136, 1997.

SANT'ANNA, G. L. et al. Módulo de resiliência de solos e misturas estabilizadas quimicamente. Porto Seguro. Anais... Porto Seguro: FOREST 2000. p. 411-413, 2000.

TRINDADE, T. P.; CARVALHO, C. A. B.; LIMA, D. C. Comportamento resiliente de um solo arenoso da região de Viçosa-MG no estado natural e estabilizado com cimento. In: REUNIÃO ANUAL DE PAVIMENTAÇÃO, 33., 2001, Florianópolis. Anais... Florianópolis: 2001. p. 369-382. 